\title{
Efficacité des relais communautaires dans le dénombrement et la détermination des populations vulnérables dans un contexte de malnutrition endémique et de conflit armé : cas d'un district sanitaire en RD Congo
}

\section{B.G. Bisimwa $a^{1,2,3}$ \\ M.T. Mambo ${ }^{2}$ \\ N.P. Mitangala $a^{2,3}$ \\ C. Schirvel ${ }^{3,4}$ \\ D. Porignon ${ }^{3,4}$ \\ W.M. Dramaix ${ }^{3,4}$ \\ P. Donnen ${ }^{3,4}$}

${ }^{1}$ Département d'épidémiologie

et de médecine sociale

École de santé publique

Université libre de Bruxelles

route de Lennik

$\mathrm{n}^{\circ} 808$

CP 595

1070 Bruxelles

Belgique

${ }^{2}$ Centre de recherche en sciences naturelles de Lwiro (CRSN)

République démocratique du Congo

${ }^{3}$ School of Public Health

Université libre de Bruxelles (ULB)

Belgique

<ghislainbiba@yahoo.fr>

${ }^{4}$ Centre scientifique et médical

Université libre de Bruxelles pour ses activités de coopération (CEMUBAC)

<philippe.donnen@ulb.ac.be>

\begin{abstract}
Résumé
Objectif: Évaluer la capacité des relais communautaires opérant dans le domaine de la nutrition à contribuer au dénombrement de la population de leurs villages respectifs sous la coordination du district sanitaire. Méthodologie: Une étude quasi expérimentale, consistant à organiser les relais communautaires en comités villageois de nutrition et à les responsabiliser dans le dénombrement de la population de leurs villages respectifs, a été mise sur pied dans un district sanitaire rural du Kivu (RD Congo). L'étude a été réalisée de mars à avril 2003 dans une situation de conflit armé. La collecte des données de la population a été assurée par les relais communautaires. Les médianes (minimum et maximum) des proportions observées par catégories d'âge ont été présentées. Résultats: Les relais ont été capables de faire un dénombrement de la population avec des résultats fiables. Les proportions de la population par catégorie d'âge observées se sont révélées similaires à celles du modèle national issu d'une enquête réalisée par des experts. Pour les relais communautaires, un pourcentage médian de 22,2\% (6,2-100\%) des ménages par village a été considéré comme vulnérables dans le contexte de l'Est de la RD Congo, nécessitant par conséquent une aide extérieure. Conclusion: Les relais communautaires peuvent contribuer à la récolte de données démographiques fiables pouvant permettre de planifier des programmes de santé publique, et le suivi de l'évolution démographique même dans une situation d'instabilité et de conflit armé.
\end{abstract}

Mots clés : relais communautaires ; population cible ; malnutrition ; insécurité alimentaire ; conflit armé ; santé publique.

\section{Abstract \\ The effectiveness of community volunteers in counting populations and assessing their nutritional vulnerability during armed conflict: District health in D.R. Congo, Central Africa}

The study assessed the ability of community volunteers, working with district health officials, to conduct a local census to count the population in their villages and assess their nutritional vulnerability. The study involved organizing community volunteers in village nutrition committee and assigning them to count the village population in a Kivu rural health district (D.R.Congo) and assess their vulnerability in terms of nutrition. The study took place in March and April 2003, during armed conflict in the region. Community volunteers supervised by district health officials collected data, presented here as median proportions (with their Max and Min), by age category. The results show that community volunteers were able to conduct this census with reliable results. The population distribution by age category was similar to the national model from a survey by

Tirés à part : P. Donnen 
experts. The community volunteers estimated a median of $22.2 \%(6.2-100 \%)$ of households in each village in the eastern DR Congo were vulnerable and required foreign aid. Community volunteers can contribute accurately to the collection of demographic data to be used in health programme planning, thus allowing these data to be followed even during instability and armed conflicts.

Key words: community volunteers; population targets; malnutrition; food insecurity; armed conflict; public health.

D ans les pays en développement, l'effectif de la population est souvent mal connu et dans la plupart des cas, il est donc difficile de définir la population cible avec précision lorsqu'on veut planifier et évaluer les programmes de santé publique. Dans ces pays, les registres de population sont souvent inexistants ou pas à jour [1]. La population du moment est déterminée sur base de données du dernier recensement auxquelles est ajouté, chaque année, un nombre de personnes déterminées par le taux d'accroissement naturel calculé par les démographes [2]. Dans des conditions normales, il est recommandé un recensement de la population tous les dix ans, mais ce délai n’est généralement pas respecté dans les pays en développement. Des crises humanitaires surviennent souvent dans ces pays en guerre caractérisés par un état d'insécurité et de déplacement continu des populations [3]. Plus le recensement est ancien, plus le risque d'erreur dans l'estimation de la population est évident, car la structure de base de la population peut être modifiée. C'est généralement le cas lorsqu'il y a des catastrophes ayant entraîné des mouvements de populations ou des dommages particuliers pour une catégorie spécifique de la population [4].

En RD Congo par exemple, le dernier recensement scientifique de la population date de plus de vingt ans (1984) [5]. Ce pays vit dans un contexte de conflit armé depuis plus de dix ans; il y a eu des mouvements de populations et beaucoup d'autres problèmes humanitaires avec d'énormes répercussions sur la structure de base des populations dans cette région des Grands Lacs [6].

Dans un contexte de crise armée prolongée et de catastrophes comme c'est le cas pour la RD Congo, identifier la population vulnérable devant bénéficier des actions humanitaires reste difficile. Selon l'Organisation mondiale de la santé (OMS), le terme vulnérable se rapporte essentiellement aux personnes qui présentent des risques plus importants de morbidité et de mortalité, du fait de leurs caractéristiques socioéconomiques ou démographiques. On cite généralement les enfants, les femmes, et particulièrement les femmes enceintes. Et dans certaines situations on y ajoute les personnes porteuses des maladies chroniques ou invalidantes, les sujets sans revenu ou d'autres groupes marginalisés vivant dans des conditions particulièrement difficiles [7].

Sur le plan alimentaire, on considère un ménage comme "vulnérable " lorsqu'il ne dispose pas de moyens suffisants pour subvenir à ses besoins alimentaires primaires (soit par une incapacité à produire, soit du fait d'une production insuffisante), c'est généralement la définition adoptée par le Programme des Nations Unies pour l'alimentation et l'agriculture (FAO) et le Programme alimentaire mondial (PAM) [8].

En cas de crise de grande ampleur ou de catastrophe humanitaire qui touche une population importante, les intervenants ont des difficultés à identifier les vrais bénéficiaires parce qu'ils sont en plus confrontés au problème de ressources qui sont généralement limitées [9].

Il est donc important de trouver une stratégie opérationnelle qui puisse permettre aux différents intervenants, notamment dans le domaine de la santé et de la nutrition, d'estimer rapidement la population cible devant bénéficier des actions humanitaires avec une certaine fiabilité.

Les relais communautaires sont des volontaires sélectionnés au niveau des villages et ont la particularité d'être bien intégrés dans leurs communautés respectives. Ils participent à la mobilisation communautaire, et connaissent particulièrement bien les membres de leur village et les moyens de subsistance de chaque ménage [31]. L'objectif de ce travail est d'évaluer la capacité des relais communautaires opérant dans le domaine de la nutrition à contribuer au dénombrement de la population susceptible de bénéficier des programmes de santé publique ainsi que des personnes vulnérables dans un contexte d'instabilité et de conflit armé.

\section{Méthodologie}

\section{Région de l'étude}

Cette étude a été réalisée de mars à avril 2003 dans le district sanitaire de Katana, et plus précisément dans le secteur de santé de Lwiro dont la population était estimée à 30000 habitants [10].

Le district sanitaire de Katana est situé dans la province du Sud Kivu. Il fait partie de l'Est de la RDC et est situé entre 1500 et 2000 mètres d'altitude. Comme toute la région de l'Est de la RDC, cette zone est instable depuis plus de dix ans à la suite d'événements de guerre qui secouent la région des Grands Lacs de l'Afrique [11].

\section{Plan et sujets de l'étude}

Il s'agit d'une étude quasi expérimentale qui consiste à suivre la mise en œuvre du programme de nutrition à assise communautaire (NAC) dans un secteur de santé composé de 31 villages répartis dans deux aires de santé (RECO). Après sensibilisation, les habitants de chaque village ont désigné cinq représentants sur la base de critères précis : motivation personnelle, dévouement pour la communauté, intégrité, moralité, emploi ou occupation, capacité à lire et à écrire. La priorité a été donnée aux femmes pour avoir au moins $60 \%$.

Des comités villageois composés de cinq membres de professions différentes ont été constitués dans chaque village et ont été responsables du dénombrement de la population de leurs villages respectifs, sous la supervision de l'infirmier titulaire du centre de santé. 
Le dénombrement s'est déroulé suivant le schéma ci-dessous :

- le dénombrement a toujours été fait en équipe (minimum trois personnes); les habitants du village étaient prévenus du jour de passage des relais ;

- les données issues du dénombrement ont été transcrites dans des registres, destinés à être conservés par les comités villageois, et sur des fiches préétablies avec des cases spécifiques par catégorie de population à retourner au bureau central ;

- le dénombrement se faisait ménage par ménage en présence du chef de ménage, généralement le soir après le retour du travail ;

- toutes les personnes en résidence temporaire au village (moins de six mois) n'ont pas été prises en compte ; par contre, les membres du village en déplacement pour une durée de moins de six mois ont été inclus dans le dénombrement ;

- chaque chef de ménage devait présenter la liste des personnes vivant sous son toit en présence de deux autres personnes majeures (dont la conjointe) ;

- l'âge des enfants de moins de cinq ans a été catégorisé en : inférieur à six mois, 6 à 11 mois, 12 à 23 mois, 24 à 59 mois. Pour les enfants de moins d'une année, l'âge était déterminé sur base d'un document mentionnant la date de naissance (carte de baptême, carnet sanitaire, carte d'identité des parents, etc.). Pour les enfants plus âgés, à défaut d'un document fiable, les enquêteurs devaient chercher à vérifier l'exactitude de la date de naissance fournie par les parents, en sollicitant les souvenirs de ces derniers par rapport aux principaux événements ayant marqué la zone (périodes de guerre, fêtes de fin d'année, fête de Pâques, etc.) ;

- pour les femmes, l'âge de procréation va de 15 à 49 ans ;

- en cas de polygamie ou de femmes vivant seules, le ménage était identifié à partir de la femme. Les enquêteurs devaient veiller à ce que les hommes polygames ne soient pas enregistrés plusieurs fois. Ainsi, était considéré comme ménage, le foyer composé d'une mère et de ses enfants ;

- était considéré comme ménage vulnérable, tout ménage avec un antécédent de malnutrition dans les deux dernières années et/ou ne disposant d'aucun mécanisme de survie d'après les autres membres de la communauté. Cette appré- ciation devait être confirmée par les voisins et le chef du village. Un terme local "bazamba " est utilisé pour désigner ce genre de ménage. La vulnérabilité est comprise dans le sens d'insécurité alimentaire. Les données en rapport avec la vulnérabilité des ménages ont été comparées à celles obtenues dans d'autres enquêtes réalisées par les autres organisations intervenant dans le domaine de nutrition et de sécurité alimentaire ;

- était considéré comme ménage sans terre, tout ménage ne disposant pas d'un lopin de terre propre (contrat foncier). Ces ménages sont sous-logés par les grands propriétaires terriens ou les chefs du village [12] ;

- à la fin du dénombrement, chaque comité villageois devait se réunir avec le chef du village, revoir les chiffres issus du dénombrement et s'assurer que tous les ménages du village avaient été pris en compte ;

- les relais communautaires ont reçu comme motivation une somme équivalente à 3 \$ américains en monnaie locale en considérant que le temps total consacré à cette activité était de deux demi-journées, soit un total déboursé de 600 \$ en y intégrant les autres petits frais du bureau du district (fiches, registres, stylos et carburant).

\section{Contrôle et gestion des données}

Dix jours après le début de l'opération, une réunion de validation des données était organisée au niveau de l'aire de santé en présence des différents comités villageois et des chefs de village. L'enquête a été supervisée par une équipe du bureau central comprenant le médecin chef de district sanitaire, un nutritionniste, un technicien en développement rural et un représentant de l'autorité chargée de l'administration publique.

\section{Méthodes statistiques}

Les données recueillies ont été codées sur ACCESS, et les analyses ont été faites avec le logiciel SPSS version 11.0. Les proportions des sujets par catégorie d'âge, le nombre de

\footnotetext{
${ }^{1}$ Bazamba est un terme utilisé dans le milieu du Bushi pour désigner un ménage considéré comme dépourvu de tout moyen de survie. Généralement, il s'agit des ménages sans terre, sans aucune autre activité génératrice de revenu, et incapables de développer un mécanisme quelconque de production alimentaire (agriculture ou élevage).
}

personnes et de ménages relevés par les relais communautaires dans chaque village ont été décrits par la médiane, accompagnée du minimum et du maximum comme mesure de dispersion. Les graphiques ont été réalisés à partir du logiciel Excel. La population dénombrée par les relais a été groupée par catégorie d'âge, et les différentes proportions ont été comparées aux différentes proportions du modèle national issu du "Multiple Indicators Cluster Survey 2002 " (enquête MICS2) [13].

\section{Complétude des rapports}

Le dénombrement a été réalisé en avril 2003 après la formation des relais communautaires. Les 31 villages ont tous remis les fiches de récolte des données dans un délai de dix jours, soit un taux de complétude de $100 \%$.

\section{Résultats}

\section{Dénombrement de la population}

Le tableau 1 compare la répartition de la population dénombrée par les relais communautaires à celle estimée à partir des résultats obtenus lors de la campagne de vaccination de masse contre la poliomyélite. On note de 8 à $9 \%$ de moins de population dénombrée par rapport à celle de référence.

\section{Présentation de la population par catégorie d'âge comparée à celle du modèle national issu de l'enquête MICS2}

Le tableau 2 démontre en particulier la plus grande faiblesse de dénombrement dans la catégorie des femmes en âge de procréer ou enceintes.

\section{Présentation de la proportion des ménages vulnérables comparée à celle des autres enquêtes}

La figure 1 démontre à l'évidence l'énorme écart observé dans la comparaison des pourcentages de ménages vulnérables selon les investigateurs. 


\section{Tableau 1. Population dénombrée et comparée à la population estimée par le district.}

Table 1. Population counted and compared to the estimated population, according to the district.

\begin{tabular}{ccc}
$\begin{array}{c}\text { Population } \\
\text { RECO }\end{array}$ & $\begin{array}{c}\text { Population de } \\
\text { référence }\end{array}$ & $\begin{array}{c}\text { Différence (\%) avec la } \\
\text { population de référence }\end{array}$ \\
\hline
\end{tabular}

Aire de santé

$\begin{array}{lrrr}\text { Buhandahanda } & 6337 & 6874 & -7,81 \\ \text { Lwiro } & 18824 & 20625 & -8,73 \\ \text { Total secteur } & 25161 & 27499 & -8,50\end{array}$

\section{Tableau 2. Répartition de la population par catégorie d'âge comparée au modèle national.}

Table 2. Population distribution according to age categories, compared with the national model.

\begin{tabular}{lrlr}
\hline Catégorie & Médiane & (min, max) & $\begin{array}{c}\text { Modèle } \\
\text { national }\end{array}$ \\
\hline Proportion d'enfants < 6 mois & $2,4 \%$ & $(0,8-6,7 \%)$ & $2 \%$ \\
Proportion d'enfants 0-59 mois & $21,3 \%$ & $(13,1-32,7 \%)$ & $18,9 \%$ \\
Proportion de femmes enceintes & $2,1 \%$ & $(0,6-5,1 \%)$ & $4 \%$ \\
Proportion de femmes en âge de procréer & $15,6 \%$ & $(3,0-25,2 \%)$ & $21 \%$ \\
Population village & 697 & $(192-1780)$ & $\mathrm{ND}$ \\
Nombre de ménages par village & 140 & $(34-332)$ & $\mathrm{ND}$ \\
Nombre de personnes par ménage & 5 & $(4-16)$ & 6 \\
Proportion de ménages vulnérables & $22,2 \%$ & $(6,2-100 \%)$ & $\mathrm{ND}$ \\
Proportion de ménages sans terre & $18,1 \%$ & $(0,9-95,7 \%)$ & $\mathrm{ND}$ \\
\hline
\end{tabular}

a Un bazamba désigne un ménage pauvre, sans aucune propriété et incapable de survivre sans une aide venant de l'extérieur (voisins ou autres).

\section{Discussion}

L'implication de relais communautaires dans le dénombrement des populations au Kivu suscite quelques points de réflexion, notamment sur la détermination de la population cible dans les cas d'urgence et l'opérationnalité des relais.

\section{Détermination de la population cible classique dans les programmes de santé}

La détermination de la population cible dans différents programmes de santé publique des pays en développement est un problème majeur. Dans la plupart des cas, il n'existe pas de données fiables sur les populations ciblées par des interventions, ce qui empêche l'évaluation précise de certains indicateurs rattachés à cette population [14]. C'est le cas de la RD Congo, où le dernier recensement scientifique date de 1984. Depuis cette date, on a seulement appliqué le taux d'accroissement démographique annuel de $2,7 \%$ [2].

Avec l'arrivée des campagnes de vaccination qui se font de porte-à-porte, le ministère se réfère aux résultats obtenus lors de ces campagnes pour déterminer la population générale, en considérant que tous les enfants de moins de cinq ans étaient atteints. Très souvent, le taux de couverture était au-delà de 100 \% dans des zones très accessibles. Deux biais sont possibles. D'abord, le retard de croissance étant fréquent dans la région, certains enfants âgés de plus de cinq ans seraient enrôlés dans la campagne pour confusion d'âge. Cela est repris dans divers rapports du Programme élargi de vaccination (PEV) [15]. Ensuite, il est possible que la structure d'âge soit modifiée à cause du climat de conflit armé et de l'instabilité des populations dans la région des
Grands Lacs. La plupart des auteurs s'accordent sur l'importance des mouvements des populations dans le Kivu $[16,17]$. Ces mouvements se sont intensifiés avec les guerres dans la région des Grands Lacs et surtout avec le climat d'insécurité, les violences sexuelles et beaucoup d'autres exactions devenues courantes à l'Est de la RD Congo [18, 19]. Dans le cadre de notre travail, les relais communautaires ont montré qu'ils étaient capables de faire un dénombrement précis de la population à partir des villages. Le tableau 1 montre que les chiffres obtenus par les relais communautaires sont assez proches de ceux du district sanitaire mais toujours inférieurs à ces derniers. Néanmoins, cette différence reste inférieure à $10 \%$. Une des explications possibles est que nous avons travaillé dans un secteur de santé où les déplacements vont dans un sens unique. En effet, la situation de conflit qui prévaut à l'est de la RDC entraîne des mouvements de populations des villages vers les villes ou les cités relativement plus sécurisées [16, 17]. On peut considérer que les relais communautaires connaissent mieux les populations habitant les villages et ont donc accès à tous les ménages du village. Certains auteurs estiment que malgré sa réussite générale, le dernier recensement scientifique de 1984 en RD Congo avait comme point faible le fait que les enquêteurs n'avaient pas atteint toutes les localités du Kivu montagneux, et même certains villages au sein des localités visitées. Il y aurait eu des obstacles liés à la nature du relief et à l'insuffisance des moyens de communication ayant pour conséquence la sous-estimation de certains groupes de population [20].

En 1992, Reynders et al. ont montré que des agents de santé formés localement étaient capables de faire un dénombrement fiable de la population dans le district sanitaire de Kirotshe au Nord Kivu [21].

Dans notre expérience, si la détermination de la population globale par les relais communautaires paraît fiable, la composition de cette dernière et la répartition de différentes catégories d'âge sont sujettes à discussions.

En effet le tableau 2 montre que les proportions obtenues pour les enfants de moins de six mois et ceux de 0 à 59 mois sont comparables à celles du modèle national issu d'une enquête réalisée par des experts deux ans auparavant. Il en est de même pour le nombre de personnes par ménage [13]. Cela constitue un argument de taille en faveur du dénombrement 


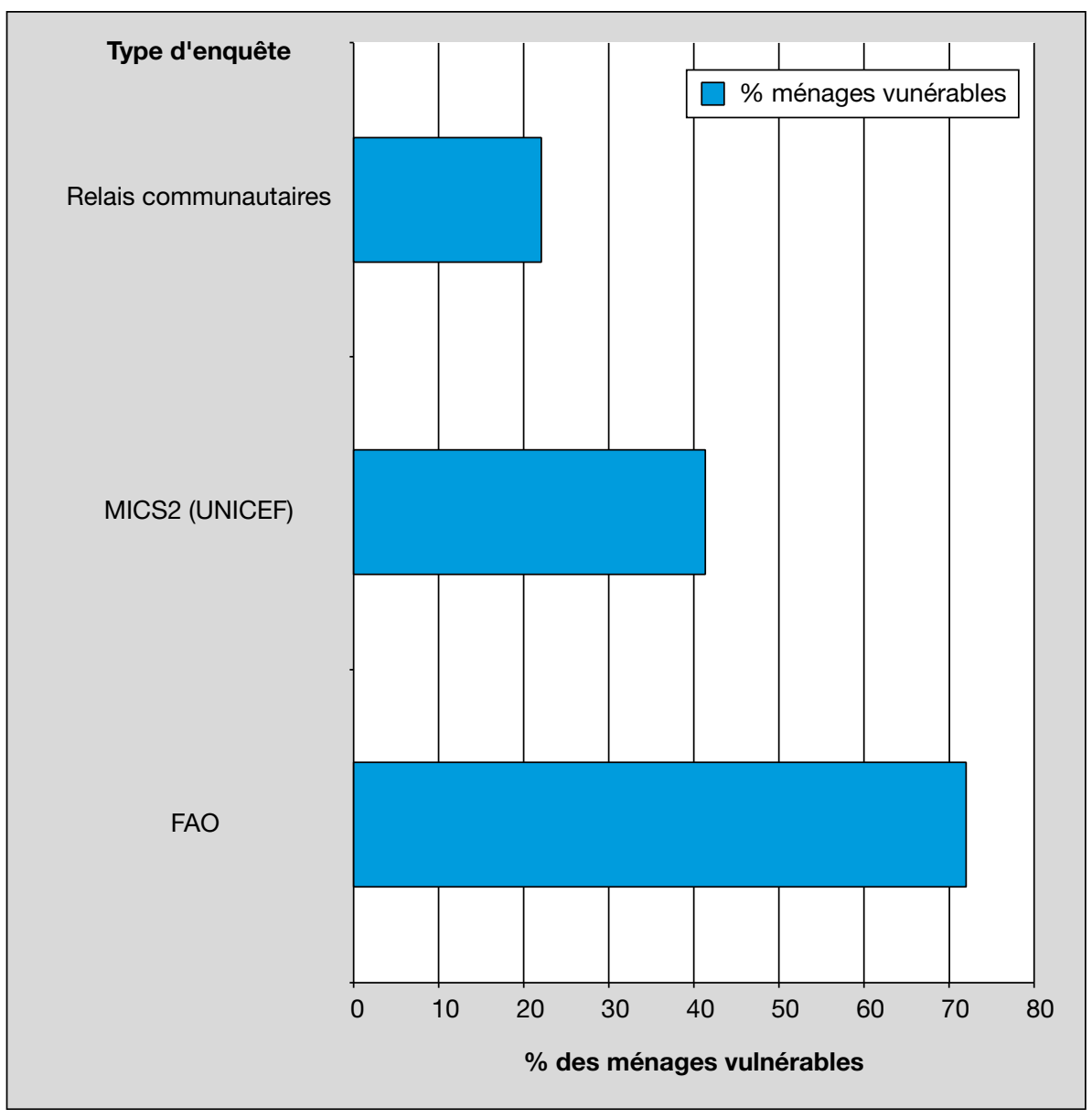

Figure 1. Degré de vulnérabilité des populations à l'Est de la RD Congo.

Figure 1. Extent of vulnerability of populations in the eastern region of Democratic Republic of the Congo.

réalisé par les relais communautaires, et c'est d'autant plus important que la plupart des interventions d'urgence visent d'abord les enfants de moins de cinq ans [22].

Le même tableau montre des écarts en ce qui concerne la proportion des femmes enceintes et des femmes en âge de procréer par rapport au modèle national, mais les proportions de ce dernier restent comprises entre les minima et les maxima des valeurs obtenues par les relais communautaires. Signalons que plusieurs auteurs ont rapporté une diminution du taux de fécondité en période de guerre, surtout lorsqu'on y associe les violences sexuelles [23, 24].

\section{Détermination des ménages " vulnérables " sur le plan alimentaire}

La terminologie vulnérable couvre plusieurs acceptations. Cependant, d'une manière générale, on s'accorde à considé- rer une population comme vulnérable lorsqu'elle présente des difficultés accrues, en termes d'intégration sociale et de survie par rapport à la population générale $[1,7,8]$. L'organisation des Nations Unies pour l'alimentation et l'agriculture (FAO) définit la vulnérabilité par rapport à l'insécurité alimentaire; un ménage est considéré comme vulnérable lorsqu'il n'est pas capable de subvenir à ses besoins alimentaires minimums [8]. C'est cette dernière définition qui a été prise en considération par les relais communautaires. Les rapports relatifs à la prise en charge de la malnutrition dans cette région montrent en effet qu'avant les événements de guerre, le taux de rechute était particulièrement élevé dans certaines familles. Pour ces dernières, certains enfants pouvaient faire trois à cinq épisodes de malnutrition aiguë sévère avant l'âge de cinq ans [25].

Dans le contexte du Kivu, les relais communautaires montrent que le pourcentage médian de ménages vulnérables par village, et donc nécessitant une aide extérieure, est de 22,2\% avec un minimum de $6,2 \%$ et un maximum de $100 \%$. Par ailleurs, on a un pourcentage médian de 18,9\% des ménages par village qui se retrouvent sans terre dans un contexte où les populations sont censées vivre d'agriculture et d'élevage. Ces résultats inquiétants peuvent justifier l'ampleur de la malnutrition dans cette région $[12,13,26]$.

La RDC figure déjà parmi les pays ayant un taux de mortalité infantojuvénile très élevé et dans ce pays, la province du Sud Kivu qui a fait l'objet de notre étude, est en tête en ce qui concerne la mortalité infantile et la prévalence de la malnutrition [12, 27, 28].

Le conflit armé qui sévit en RD Congo depuis plus de dix ans fait partie des grandes crises survenues depuis la deuxième guerre mondiale, et serait l'une des plus meurtrières avec quatre millions de décès au cours des six dernières années [29, 30].

Devant l'impossibilité à mobiliser des ressources permettant aux organisations humanitaires de faire des interventions à large échelle dans des grandes régions, il paraît donc plus efficient de mettre au point des stratégies permettant l'identification rapide des couches les plus vulnérables dans des communautés en crise.

\section{Opérationnalité des relais communautaires et leur encadrement à long terme}

Dans le contexte du Kivu, les relais communautaires ont été recrutés au sein des villages sur base des critères dont les plus importants sont la stabilité dans la communauté et le dévouement pour celle-ci. La motivation financière qui leur a été offerte est peu importante par rapport au salaire d'un agent extérieur dont les tarifs varient entre 20 à $50 \$$ par jour sans compter les frais logistiques. De plus, la collaboration avec les relais communautaires offre une bonne garantie dans la détermination des ménages vulnérables. La prise en charge de ces derniers étant généralement assurée par les voisins au nom de la solidarité africaine, toute la communauté souhaiterait qu'on trouve des solutions efficaces pour ces ménages. La coordination des activités des relais par le district sanitaire et l'intégration de ces derniers dans le système de santé peut 
garantir à la fois la qualité de leur travail et leur opérationnalité à moyen et long terme.

\section{Conclusion}

Notre travail a montré que les relais communautaires, constitués en comités villageois et dont le recrutement est basé sur l'appartenance au milieu avec une certaine hétérogénéité professionnelle, peuvent constituer un support important pour le système de santé.

Ils peuvent contribuer à la récolte de données démographiques fiables pouvant permettre la planification des programmes de santé publique ainsi que le suivi de l'évolution démographique, même dans une situation d'instabilité et de conflit armé. Leur contribution peut être utile en contexte stable ou dans une situation d'urgence pour déterminer les populations vulnérables. Un important travail mérite d'être réalisé pour apprécier de façon objective la limite de leur contribution par rapport aux informations de base à fournir pour la planification des interventions d'urgence, leur intégration dans le système de santé de base et leur opérationnalité à long terme. Leur contribution dans des programmes de surveillance épidémiologique à base communautaire constitue une nouvelle piste pour les pays en développement où la couverture sanitaire reste faible.

\section{Références}

1. WHO. The World Health Report 2005. Avri 2005. www.who.into/whr/2005 (septembre 2007).

2. Institut national de la statistique 1993. République du Zaïre, Kinshasa, Recensemen scientifique de la population, Juillet 1984 Projections démographiques : Zaïre et régions 1984-2000.
3. Van Herp $M$, Parqué V, Rackley $\mathrm{E}$, et al. Mortality, vilolence and lack of access to healthcare in the Democratic Republic of Congo. Disasters. 2003; 27: 141-53.

4. Kalipeni E, Oppong J. The refugee crisis in Africa and implications for health and disease: a political ecology approach. Soc Sci Med 1998; 46: 1637-53.

5. Institut national de la statistique 1984. Combien sommes nous? République du Zaïre, département du Plan, Kinshasa, Recensement scientifique de la population, $1^{\text {er }}$ Juillet 1984, Résultats provisoires, $67 p$.

6. WHO, Democratic Republic of Congo: a forgotten humanitarian disaster. www.who/int/ hac/donorinf/campaing.

7. Unicef. Situation des enfants dans le monde, rapport 2004-2006.

8. FAO. L'état de I'insécurité alimentaire dans le monde, Rapport SAM 2006. www.fao.org/ docrep/009/a0750f/a0750f00.htm. (septembre 2007).

9. Prudhon C. La malnutrition en situation de crise. Éditions Karthala et Action contre la faim, 2001

10. Inspection provinciale de la santé, rapports d'activités de la zone de santé de Katana, 1994-2003.

11. Porignon D. Adéquation des systèmes de santé de district en situation critique; expériences dans la région des Grands Lacs africains. Thèse présentée en vue de l'obtention du titre de Docteur en Sciences de la santé publique. École de santé publique; Université libre de bruxelles. 2003.

12. Vis $\mathrm{HL}$, Tondeur G, Carael M, Willis $\mathrm{W}$, et al. Le Kivu montagneux, Surpopulation-Sous-nutrition-Erosion du sol (Étude prospective par simulations mathématiques). Académie royale des sciences d'Outre-Mer. Classe des sciences naturelles et médicales. Mémoires in-8, Nouvelle Série, Tome 21, fascicule 3, Bruxelles, 1986

13. Unicef. Enquête nationale sur la situation des enfants et des femmes MICS 2/2001. Kinshasa, 2002 Juillet : 123-35.

14. D'Altilia JP, De Lamalle JP, De Caluwé, et al. Système $d^{\prime}$ information sanitaire. $2^{\mathrm{e}}$ Édition. L'Harmattan. 2005.

15. Ministère de la santé publique. RD Congo, Rapport d'activités du Programme élargi de vaccination (PEV) 2000-2005.

16. Mafikiri Tsongo A. Mouvements de population, accès à la terre et question de la nationalité au Kivu (Zaïre). In: Mathieu P, Laurent P-J, Willame J-C Démocratie, Enjeux fonciers et pratiques locales en Afrique, conflits, gouvernance et turbulences en Afrique de I'Ouest et centrale. Cahiers Africains, Institut AfricainCEDAF, Bruxelles, L'Harmattan, Paris, (23-24): 180-201, 1996.
17. Nicolaï H. Répartition de la population et problèmes démographiques du Kivu d'altitude. In: CEMUBAC, Actes du cinquantenaire du CEMUBAC, Bruxelles, pp 155-167, 1988.

18. UNFPA et RD Congo. Initiative conjointe de lutte contre les violences sexuelles, 2004.

19. Ministère de la condition féminine et famille, RD Congo. Rapport sur l'exploitation sexuelle des enfants. Rapport Pays, Juillet 2004.

20. Nicolaï H. La répartition et la densité de la population au Kivu pp 13-17. Académie royale des sciences d'outre-mer. Classe des sciences naturelles et médicales. Mémoire in-8, Nouvelle Série, Tome 24, fascicule 2, Bruxelles, 1998.

21. Reynders D, Tonglet R, Lembo EM, et al. Community health workers are capable of determining reliably the target population for health programs. Ann Soc Belg Med Trop 1992; 72: 145-54.

22. Guha-Sapir D, Panhuis WG. Conflict-related mortality: an analysis of 37 datasets. Disasters. 2004; 28: 418-28.

23. UNFPA. The impact of conflict on women and girls. Bratislava, Slovakia 13-15 November 2002 .

24. WHO, Gender, based violence: healing wounds in the Democratic Republic of The Congo. www.who.int. (septembre 2007).

25. Centre de recherche en sciences naturelles de Lwiro (CRSN). Rapports d'activités du département de nutrition (hôpital pédiatrique de Lwiro), 1985-2005.

26. Inspection provinciale de la santé du Sud Kivu/CEMUBAC-IRC. La sécurité alimentaire dans la zone de santé de rurale de Kabare, Enquête réalisée en collaboration avec I'Inspection provinciale de la santé ; 2002.

27. Black R, Morris Aul S, Bryce J. Where and why are 10 millions children's dying every year? Lancet 2003 June 28; 2226-34.

28. Bryce J, Boschi-Pinto C, Shibuya K, et al. WHO Child Health Epidemiology Reference Group. WHO estimates of the causes of death in children? Lancet 2005; 365: 1147-52.

29. Coghlan B, Brennan RJ, Ngoy $P$, et al. Mortality in the Democratic republic of Congo: a nationwide survey. Lancet 2006 $367: 44-51$

30. Guha-Sapir D, van Panhuis WG. The importance of conflict-related mortality in civilian populations. Lancet 2003; 361: 2126-8.

31. Bisimwa B, Dramaix W, Donnen $P$, et al Effectiveness of community volunteers in monitoring children's growth in an endemic context of malnutrition and conflict in Democratic Republic of Congo. The first congress of public health nutrition and VII congress of the Spanish Community Nutrition Society (SENC): Barcelone, September 2006). 